\title{
A Smartphone Based Ophthalmoscope
}

\author{
Mario E. Giardini-EMBS Member, Iain A. T. Livingstone, Stewart Jordan, Nigel M. Bolster, Tunde \\ Peto, Matthew Burton, Andrew Bastawrous
}

\begin{abstract}
A low-cost alternative to the direct ophthalmoscope, a simple optical adapter for a smartphone, is described. It can overcome many of the technical challenges of fundoscopy, providing a high-resolution view of the retina through an un-dilated pupil. This can be used in locations with limited diagnostic resources to detect conditions such as glaucomatous optic neuropathy. Comparison of optic nerve images from commercial retinal screening cameras with the smartphone adapter demonstrates strong evidence for no difference in performance in glaucomatous disc grading $(p=0.98$, paired student $t$ test, $n=300)$.
\end{abstract}

\section{INTRODUCTION}

In 2010, the World Health Organization estimated that 285 million people worldwide exhibit some form of visual impairment [1]. 39 million are blind and, of these, $80 \%$ can be avoided or cured. Yet, $90 \%$ of blind people live in lowincome countries, where the critical limiting factor in providing treatment is the low number of practicing ophthalmologists. Indeed there is an inverse relationship between the prevalence of blindness and the number of ophthalmic care providers within reasonable reach. [2].

In the diagnostic chain necessary to secure ophthalmic treatment, the examination of the retina through the eye's pupil (ophthalmoscopy) is a key diagnostic requirement [3]. Through ophthalmoscopy it is possible to screen for the patients who would need further specialist attention. Ideally, such screening should happen in the community, as to optimize the referrals to the highly scarce specialists, minimizing unnecessary referrals. Yet, current ophthalmoscopes rely on optical designs developed in the early twentieth century [4], and the level of specialist skills required for their use severely limits their applicability by untrained personnel or by practitioners making infrequent

Research supported by the Medical Research Council, UK, and The Queen Elizabeth Diamond Jubilee Trust, UK.

Mario E. Giardini and Nigel M. Bolster are with the Department of Biomedical Engineering, University of Strathclyde, Glasgow G4 0NW, UK (M.E.Giardini corresponding author - phone: +44-141-5483042; fax: +44141-5526098; e-mail: mario.giardini@strath.ac.uk - N.M.B. email nigel.mycock@start.ac.uk)

Iain A. T. Livingstone is with Glasgow Centre for Ophthalmic Research, Tennent Institute of Ophthalmology, Greater Glasgow \& Clyde. Gartnavel General Hospital, Glasgow G12 0YN (e-mail: iain@peekvision.org)

Tunde Peto is with NIHR Biomedical Research Centre at Moorfields Eye Hospital NHS Foundation Trust and UCL Institute of Ophthalmology, 162 City Road, London EC1V 2PD, UK (e-mail: Tunde.Peto @)moorfields.nhs.uk)

Andrew Bastawrous, Stewart Jordan and Matthew Burton are with the International Centre for Eye Health (ICEH), Clinical Research Department, Faculty of Infectious \& Tropical Diseases, London School of Hygiene \& Tropical Medicine, Keppel Street, London WC1E 7HT, UK (e-mails: andrew.bastawrous@1shtm.ac.uk, stew@peekvision.org, Matthew.Burton (a) 1shtm.ac.uk) use. $[5,6]$. As a consequence, in low-income countries the lack of skilled personnel ultimately impacts on the delivery of ophthalmoscopy services. Therefore, an ophthalmoscope which can be used by personnel with minimal training is strongly and urgently needed.

The implications for a mobile-based equivalent to the direct ophthalmoscope are far-reaching. For the purposes of validating the device in the present article, a condition with gradable features allows objective comparison with traditional imaging techniques. Glaucoma is a blinding condition characterized by a loss of the optic nerve neuroretinal rim, with associated visual field compromise. Clinically, the "cup:disc ratio" provides a visible signpost of glaucomatous optic nerve damage, which is graded on visual inspection of the retina. The cup:disc ratio is a continuum; 0.1 represents a healthy optic disc, without glaucomatous loss of the neuroretinal rim. A cup:disc ratio of 1.0 represents absolute disc cupping, typically associated with visual field wipeout, and blindness.

In the present paper we describe the design of a smartphone-based ophthalmoscopy solution dedicated to infield screening in low- and middle-income countries. This solution has undergone extensive data collection in field studies in Kenya (Nakuru Eye Study) within the project "Peek - Portable Eye Examination Kit" [7]. We present preliminary performance data measured on patients undergoing screening for glaucomatous optic neuropathy.

\section{METHODS}

\section{A. Smartphone and ophthalmoscope}

The mobile phone platform chosen has been the Samsung Galaxy S III (Samsung, South Korea). This smartphone was chosen as the best compromise at the time of conception (2012), balancing cost, good quality back-facing camera, sufficient screen size, and the use of an Android operating system, for best inter-platform compatibility.

An ophthalmoscopy adapter has been designed and attached to the smartphone, as per Figure 1. The adapter is composed of a plastic shell that attaches to the back of the phone, akin to a common phone cover, carrying a singlepiece custom plastic optical assembly. The device is entirely manufacturable through a standard injection molding process, presents no critical alignments, and therefore lends itself to mass production at costs compatible with distribution to community healthcare operators. On the adapter, the phone camera flash (white LED) is deflected through a the optical assembly in order to align and match the illumination path with the field of view of the camera. A metal shield screens the camera lens from the light 
backscattered by the prism surface imperfections. The prism and the shield are mounted on a 3D-printed (PP3DP Up Plus 2, China) black plastic (polylactic acid, PLA) clip that holds them in alignment in front of the phone flash and camera.

In order to acquire images of the retina, the phone camera is held close to front of the eye. For the examinations described in this paper, the pupils were dilated (Guttae Tropicamide $1 \%$ ), though the adapter has been designed to offer high-resolution disc imaging through an undilated pupil, with readily gradable disc images attained from $3 \mathrm{~mm}$ pupils. The adapter is operated by turning the phone flash on, and by putting the phone in video recording mode, either through its own video recorder, or through an ophthalmology-dedicated software, as described below. A stream of video frames is recorded, each frame effectively representing an image of the fundus, as per the following paragraph. The typical viewing angle is thirty degrees.

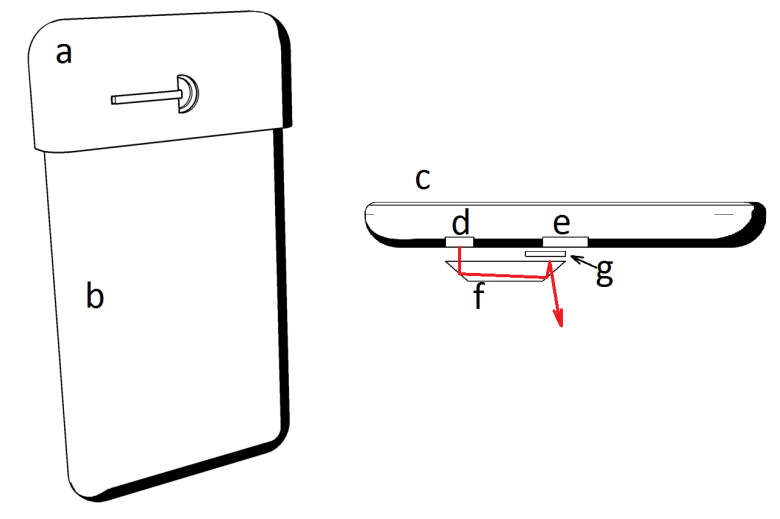

Figure 1. Ophthalmoscope adapter. (Left: adapter (a) assembled on phone (b). Right: (c) phone, (d) flash LED, (e) camera, (f) prism, (g) shield, (red arrow) light path)

\section{B. Smartphone software}

In the field study, the fundus images have been acquired through dedicated software running on the smartphone. The software has been implemented in Java, as per common practice under the Android operating system 4.2 [9]. Using such software, the operator starts a video recording on the phone. The phone flash turns on, and light is provided to the ophthalmoscope. Once a brief video sequence containing images of the fundus has been captured, the operator turns the video recording off. The video is then manually clipped to extract the segment effectively visualizing the fundus, and a frame in which the optic disc is clearly visible is manually selected. Finally, through drag-and-pinch motions on the phone screen, the operator manually zooms onto the optic disc, and sets two cursors on a full vertical disc diameter and on the vertical cup diameter. The software determines the ratio between the two cursor lengths (cup:disc ratio), which is stored alongside patient identification data for further analysis. Furthermore, the software allows transmission of the images remotely, for grading, archiving and second opinions in cases of diagnostic doubt.

\section{Field data and analysis}

The Nakuru Eye Disease Cohort Study [8] formed the control arm of the comparative study. In the cohort study, all participants underwent retinal imaging with a Digital Retinal Camera (DRS+, Haag Streit CenterVue, Italy) by an ophthalmic assistant. The same particpants were separately examined using the smartphone based ophthalmoscope. Images were independently graded at Moorfields Eye Hospital, London, where a masked grader graded retinal images from both systems. Included in the grading was the vertical optic disc to cup ratio (VCDR).

Interim VCDR results measured on 300 eyes using both systems are reported.

\section{RESULTS}

An example image of the fundus, captured in an adult male volunteer is reported in Figure 2. As per typical use in the study, the image has been captured by an operator with no ophthalmology background, who has been given a few minutes induction on the device only.

Field studies are in progress, with data collected for $>1000$ eyes. Interim analysis of a 300 eye subset evidences no statistically significant difference in VCDR between dilated DRS fundus photography and smartphone-acquired footage $(p=0.98, N=300)$. VCDR ranged from 0.1 to 1.0 in the subgroup.

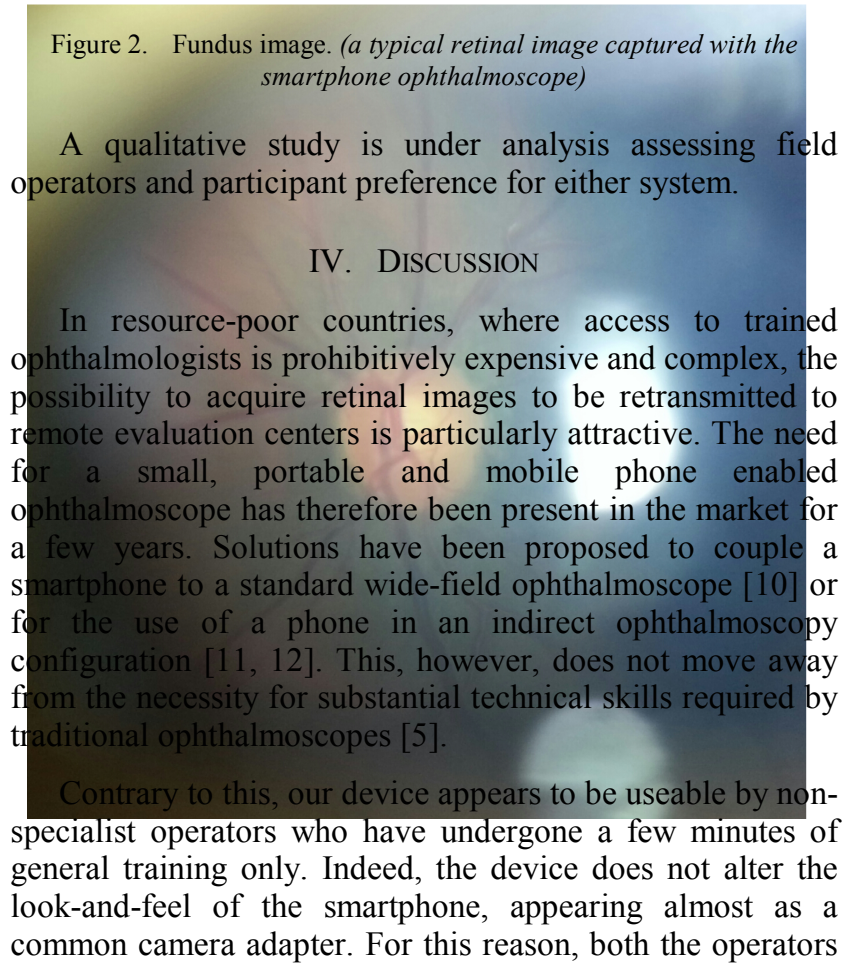


and the patients appear not to be intimidated by the technology, and accept their use instinctively.

In this respect, the software has been designed as to involve operations (video capture, drag, pinch-and-zoom motions) with which smartphone-literate operators are familiar. To the operators, the whole acquisition and measurement process appears as an overlay to a common video/snapshot acquisition. In contrast to traditional techniques, the digital acquisition of images allows dynamic digital overlays, guiding disc-grading through manipulation of digital measuring cursors. With drag/pinch/zoom functionality, an objective measure of disc and cup dimensions is possible. With minimal training, reproducible grading can be performed.

Moreover, unlike current smartphone/ophthalmoscope adapters that couple the smartphone optic to a conventional ophthalmoscope, the present system entirely avoids the presence of a direct ophthalmoscope. Harnessing the autofocussing properties of the native smartphone camera, the cumbersome focusing wheel central to the traditional device is entirely unnecessary, markedly increasing ease of use. In addition, the smartphone solution can be held at arms length, in contrast to traditional means, whereby the examiners eye and face are required to be within $5 \mathrm{~cm}$ of the patient's eye. The increased working distance increases examination comfort, and offers a less intimidating experience for pediatric patients.

The formal image analysis requires traceable and recorded objective imaging. For this reason, the validation is being pursued by comparison with the DRS camera which, for our intended use, we can assume to perform better than the direct ophthalmoscope, as it allows a formal measurement of the relevant distances [13].

The interim results are strongly suggestive that the image quality obtained using the smartphone adapter is fit for glaucomatous disc grading. Moreover, the field aperture compares favorably to what attainable using current smartphone-to- ophthalmoscope coupling techniques, reaching values of approximately 30 degrees. The resolution and frame-rate are dependent on the specifications of the smartphone platform. For our Samsung S III platform, video footage comprises 30fps, with HD video resolution (1920x 1080 pixels, 1080p standard), allowing detailed fundus examination from the extracted frames.

Importantly, a recent review of smartphone software for eye care use has highlighted that, although a significant number of apps are available, the vast majority lack the validation necessary for use in clinical settings [14] Contrary to this, our system appears to be amongst the minority where substantial quantitative and qualitative validation data are available, with continued validation study in progress.

It is important to underline the device is intended to be used for screening and monitoring within the community of patients at risk, with the potential for data to be forwarded to local specialists for grading. This finds applications in systemic conditions that have eye manifestations which can act as a marker of disease, e.g. Malaria Retinopathy in cerebral malaria, and in pathologies that can result in sight loss if not managed early in the disease process, e.g Diabetic Retinopathy.

\section{CONCLUSION}

In this paper, we have described our design and implementation of a smartphone adapter and software to perform imaging of the retina, and shown preliminary validation results on glaucomatous optic retinopathy. We expect the vast amount of data collected during the Peek field studies in Kenya to yield further important validation information, and to inform the design parameters for fieldoptimized developments of the adapter and software, with the ultimate target of delivering an ophthalmoscopy toolkit for better eye care in low- and middle-income countries.

While the device was designed with a focus on usage in low income settings, the key features of ease of use, portability and low cost make it attractive for use in high-income environments. It can significantly extend the range of high quality retinal imaging in any situation, allowing for off-site screening, remote diagnostic assistance and significantly reduced costs to healthcare systems. Further developments will also be targeted towards this direction.

\section{APPENDIX}

The study adhered to the tenets of the Declaration of Helsinki and was approved by the Ethics Committee of London School of Hygiene and Tropical Medicine and the African Medical and Research Foundation (AMREF), Kenya. Approval was also granted by the Rift Valley Provincial Medical Officer and the Nakuru district Medical Officer of Health. Approval was sought from the administrative heads in each study cluster.

Intellectual property aspects from this article are protected by Peek [6].

\section{ACKNOWLEDGMENT}

We wish to thank the field team of the Nakuru Eye Study.

\section{REFERENCES}

[1] Fact Sheet $\mathrm{N}^{\circ} 282$ "Visual impairment and blindness", World Health Organization, 2010

[2] A Bastawrous, B. D. Hennig, "The Global Inverse Care Law: A Distorted Map of Blindness," British Journal of Ophthalmology vol. 96 pp. $1357-1358,2012$

[3] "Comprehensive Adult Medical Eye Evaluation", Preferred Practice Pattern Guidelines, American Academy of Ophthalmology, 2010

[4] C. R. Keeler, "A Brief History of the Ophthalmoscope", Optometry in Practice vol. 4 pp. 137-145, 2003

[5] E. Roberts, R. Morgan, D. King and L. Clerkin, "Funduscopy: a forgotten art?", Postgraduate Medical Journal vol. 75 pp. 282-284, 1999

[6] S. Dalay, F. Umar and S. Saeed, "Fundoscopy: a reflection upon medical training?" Clinical Teacher vol. 10 pp. 103-106, 2013

[7] www.peekvision.org, last visited on 2 April 2014. 
[8] A. Bastawrous, W. Mathenge, T. Peto, H. A. Weiss, H. Rono, A. Foster, M. Burton, H. Kuper, "The Nakuru eye disease cohort study: methodology \& rationale”, BMC Ophthalmology vol. 14 art. 60, 2014

[9] M. L. Murphy, The busy coder's guide to Android development. CommonsWare LLC, 2008, pp. xv-xvi

[10] http://www.welchallyn.com/promotions/iExaminer/index.html, last visited on 4 April 2014

[11] A. Bastawrous, "Smartphone Fundoscopy" Ophthalmology vol. 119 pp. 432-433, 2012

[12] D. Myung, A. Jais, L. He, M. S. Blumenkranz, R. T. Chang, "ED Printed Smartphone Indirect Lens Adapter for Rapid, High Quality Retinal Imaging", Journal of Mobile Technology in Medicine vol. 3 pp. 9-15, 2014

[13] R. Bernardes, P. Serranho and C. Lobo "Digital Ocular Fundus Imaging: A Review", Ophthalmologica vol. 226 pp. 161-181, 2011

[14] A.Bastawrous, R. C. Cheeseman and A. Kumar, "iPhones for eye surgeons", Eye vol. 26 pp. 343-354, 2012 